\title{
Simultaneous Golgi-Cox and immunofluorescence using confocal microscopy
}

\author{
Saturnino Spiga $\cdot$ Elio Acquas $\cdot$ Maria C. Puddu • \\ Giovanna Mulas $\cdot$ Alessandra Lintas • \\ Marco Diana
}

Received: 4 June 2010/Accepted: 17 March 2011/Published online: 2 April 2011

(C) The Author(s) 2011. This article is published with open access at Springerlink.com

\begin{abstract}
Visualization of neuronal elements is of fundamental importance in modern neuroscience. Golgi-Cox impregnation is a widely employed method that provides detailed information about morphological characteristics of neurons, but none regarding their neurochemical features. Immunocytochemical procedures, on the other hand, can provide a high degree of biochemical specificity but poorer morphological details, in particular if compared to GolgiCox impregnation. Hence, the combined use of these two approaches is highly desirable, especially for confocal microscopy that can exploit the advantages of both methods simultaneously. Here we show an innovative procedure of perfusion and fixation of brain tissue, that allows, by applying Golgi-Cox impregnation and immunofluorescence in the same histological section, to obtain high-quality histological material, with a very simple and inexpensive method. This procedure is based on three simple fixation steps: (1) a paraformaldehyde perfusion followed by a
\end{abstract}

\footnotetext{
S. Spiga $(\bowtie) \cdot$ M. C. Puddu · G. Mulas

Department of Animal Biology and Ecology,

University of Cagliari, Via Ing. Tomaso Fiorelli,

09126 Cagliari, Italy

e-mail: sspiga@unica.it

E. Acquas

Department of Toxicology, INN-National Institute

of Neuroscience, Center of Excellence

on Neurobiology of Addiction, 72, 09124 Cagliari, Italy

A. Lintas

Departement of Medicine/Unit of Anatomy, University of

Fribourg, Rte Albert Gockel 1, 1700 Fribourg, Switzerland

M. Diana

"G.Minardi" Laboratory of Cognitive Neuroscience, Department of Drug Sciences, University of Sassari, via Muroni 23, 07100 Sassari, Italy
}

standard post-fixation to stabilize the subsequent immunofluorescence reaction; (2) the classical Golgi-Cox impregnation and (3) an immunofluorescence reaction in previously impregnated material. This combination allows simultaneous visualization of (a) the structural details (Golgi-Cox impregnated neurons), (b) the antigens' characterization, (c) the anatomical interactions between discrete neuronal elements and (d) the 3D reconstruction and modeling. The method is easy to perform and can be reproducibly applied by small laboratories and expanded through the use of different antibodies. Overall, the method presented in this study offers an innovative and powerful approach to study the nervous system, especially by using confocal microscopy.

Keywords Golgi-Cox · Immunofluorescence · PSD-95 · Synapsin I - Tyrosine hydroxylase · Confocal microscopy

\section{Introduction}

Few histological techniques have played a significant role in understanding the nervous tissue as Golgi methods (Speacek 1989). The Golgi's impregnation was originally proposed by Golgi (1873) with the application of potassium dichromate followed by silver nitrate to obtain its famous "reazione nera" (black reaction). Cox (1891) modified this method by treating the tissue with a potassium dichromate and mercuric chloride solution. The principle of these reactions is based on the black deposit in impregnated nerve cells, which have been submitted to alkalinization (Stean 1974) to produce mercurous chloride $\left(\mathrm{Hg}_{2} \mathrm{Cl}_{2}\right)$, topographically associated exclusively within stained structures (Fregerslev et al. 1971) and presumably following the formation of microcrystals localized within the thickness of the plasma membrane. These elements appear dark in a very clear background 
(Buell 1982) fully and finely delineated, but the most intriguing, still unresolved feature, is that their impregnation is by chance, staining fewer than $5 \%$ of the neuronal population exposed (Speacek 1989).

The Golgi-Cox method, since its discovery, has been usefully applied for qualitative analysis of neuronal morphology and quantitative evaluations such as dendritic spine counts (Robinson and Kolb 1999; Spiga et al. 2005; Diana et al. 2006; Sarti et al. 2007; Flores et al. 2007; Kasture et al. 2009), dendritic length measurements and branching complexity (Kolb et al. 2008; Rema et al. 2008). This method is very suitable using confocal microscopy, in reflection modality, for modeling and 3D reconstructions (Spiga et al. 2005). A major advantage of confocal microscopy in reflection modality is the ability to image metallic impregnated tissues of a wide variety, including brain, muscular fibers and skin. Metallic grains are capable to reflect the laser light and are completely indifferent to the photobleaching phenomenon. Probes that can be used in reflected light mode include gold particles, peroxidase labels, and silver grains.

The success of the immunohistochemical procedures is dependent upon the ability of antibodies to bind specific antigens with high affinity and it may be used to localize antigens at the cellular and subcellular level. The extraordinary explosion in popularity of confocal laser scanning microscopy (CLSM) in recent years, is due, in part, to the relative ease with which extremely high-quality images can be obtained from specimens prepared for conventional light (impregnation) or fluorescent microscopy (Muller 2002). In fact, the CLSM offers several advantages compared to conventional widefield optical epi-fluorescence microscopy, including the ability to control the depth of field, the reduction of background noise and the capability to collect serial optical sections from specimens. Furthermore, by suppressing out-of-focus light, the CLSM, allows the detection of fine details that might represent precious information that would be lost in conventional microscopy.

In the past, some attempts have been made to combine the "reazione nera" with the immunoperoxidase procedure. In the early $80 \mathrm{~s}$, very complicated procedures were proposed by Somogyi et al. (1983) in cat's visual cortex and by Freund and Somogyi (1983) in monkey's visual cortex and retina in order to combine Golgi impregnation with histochemistry, after horseradish peroxidase transport. Freund and colleagues (1984) and Somogyi (1990) further extended the method to electron microscopy, whereas Buller and Rossi (1993) tested a large number of antibodies in combination with different approaches (de-impregnation). However, albeit with somehow good results, these efforts have been frustrated by the high degree of complexity and low reproducibility of such methodological procedures (Somogyi et al. 1983; Freund and Somogyi 1983; Somogyi 1990; Buller and Rossi 1993).
In recent years, many perfusion and fixation procedures have been proposed to render the Golgi method (and its numerous variants) more versatile and adapt to different intents. For instance, tissues have been fixed in formalin for variable times, spanning from few hours in Buell (1982) and Williams (1983), to several years (Glantz and Lewis 2000; Rosoklija et al. 2000) up to 55(!) (Gorazd et al. 2003). Similar attempts were also made for Golgi applications to electron microscopy called Golgi-Kopsch (Kopsch 1896) and Colonnier-Golgi (Colonnier 1964). Interestingly, to our knowledge, immunofluorescent double-labeling was never described in combination with Golgi-Cox impregnation, possibly because it is generally considered not compatible with the "black reaction" due to significant differences in the perfusion and fixation procedures of the two methods (Lee et al. 2006).

The development of the method described in the present study was motivated by the serendipitous intuition that the combination of three key elements, namely the Golgi-Cox impregnation, the immunofluorence and the CLSM, might synergistically interact to provide a method able to greatly amplify the individual characteristics of each method taken separately. In particular, the method presented in this work allows to circumvent the above-described methodological constraints of the previous attempts to combine the Golgi and immunoperoxidase methods by profiting of the high resolution of CLSM. The proposed method was tested in the dopamine (DA) containing pathway that has been previously investigated separately (Spiga et al. 2003; Spiga et al. 2005). Thus, several brain areas were examined from mesencephalic structures to striatum and medial prefrontal cortex (PFC). Three antigens were marked: Tyrosine Hydroxylase (TH) to detect presumably DAergic neuronal elements, Synapsin I (SynI) and Post-synaptic density 95 (PSD-95) to visualize pre and post-synaptic proteins, respectively. The results demonstrate that immunofluorescence, applied to Golgi-Cox stained material, is a method (1) simple and unexpensive; (2) highly reproducible, even in standard laboratory set-up and (3) allows the simultaneous visualization of biochemically characterized impregnated neuronal elements. In addition, the application of CLSM allows to obtain very detailed and explicative pictures. Finally, our results suggest that this method can be applied to a wide variety of different antibodies and/or tracers related to virtually any nervous system-related research need.

\section{Methods}

Experimental procedures

Twenty-two male Sprague-Dawley albino rats weighing 200-225 g Charles River (Como, Italy) were used. 
Experimental protocols were performed in strict accordance with the E.C. regulations for the use of experimental animals (CEE NO. 86/609) and recommended guidelines for the care and use of experimental animals approved by the Society for Neuroscience (USA).

\section{Golgi-Cox procedure}

Animals were deeply anesthetized with chloral hydrate and perfused intracardially with $0.9 \%$ saline solution $(400 \mathrm{ml})$ followed by $4 \%$ paraformaldehyde $(\mathrm{pH} 7.4)$ (200 ml). Brains were carefully removed from skull and postfixed in same fixative overnight at $4^{\circ} \mathrm{C}$. After, were washed in 0.4 M Sorenson's Phosphate Buffer (PBS) at least for $2 \mathrm{~h}$ (three changes, $20 \mathrm{~min}$ each) to remove any trace of fixative and placed in $20 \mathrm{ml}$ of Golgi-Cox solution. The composition of Golgi-Cox solution was: $5 \%$ potassium dichromate, $5 \%$ mercuric chloride, $5 \%$ potassium chromate (pH 6.5) (Adermark and Lovinger 2006). The brains remained immersed in this solution and after 2 days were immersed in an identical volume of fresh Golgi-Cox solution for additional 14 days in the dark to reduce background staining. The brains were then transferred in a $30 \%$ sucrose solution in PBS for cryoprotection for 2-3 days. Afterward, the brains were cut in $100 \mu \mathrm{m}$ thick coronal slices using a cryostat (Microm Cryo-Star HM 560, Walldorf, Germany). To prevent section cracking the brains and knife were kept at $-3^{\circ} \mathrm{C}$ and $-13^{\circ} \mathrm{C}$, respectively. Slices were developed using the procedure described by Kolb and McClimans (1986).

\section{Immunofluorescence procedure}

At the end of the Golgi-Cox procedure indicated above, slices were not dehydrated and mounted as usual, but collected in PB for the following free floating immunostaining.

Slices were rinsed in PBS $(3 \times 10 \mathrm{~min})$. To prevent non-specific binding, slices were pre-incubated in 5\% normal goat serum (NGS) containing $5 \%$ bovine serum albumin (BSA) and $0.5 \%$ Triton X-100 in PBS overnight at $4^{\circ} \mathrm{C}$.

Four primary antibodies were used:

polyclonal rabbit anti-TH (Santa Cruz Biotechnology, Inc),

monoclonal mouse anti-TH (Sigma-Aldrich), polyclonal rabbit anti-SynI (Santa Cruz Biotechnology, Inc),

monoclonal mouse anti PSD-95 (Santa Cruz Biotechnology, Inc).

Slices were then incubated with primary antibodies in four combinations of protocols:
Only mouse anti-TH (A); Rabbit Anti-TH and mouse anti PSD-95 (B); mouse anti-TH and rabbit anti-SynI (C); rabbit anti-SynI and mouse anti PSD-95 (D).

A: Mouse anti-TH (1:400) in PBS for $48 \mathrm{~h}$ at $4^{\circ} \mathrm{C}$. Following $3 \times 10$ min washes in PBS, sections were incubated in biotinylated goat anti-mouse secondary antibody (1:200, Vector Laboratories, Burlingame, CA) in PBS for $2 \mathrm{~h}$ at room temperature (RT), washed $3 \times 10 \mathrm{~min}$ in PBS, and incubated in FluoresceinStreptavidin (1:200, Vector Laboratories, Burlingame, $\mathrm{CA}$ ) in PBS for $2 \mathrm{~h}$ at $\mathrm{RT}$

B: cocktail of rabbit Anti-TH (1:1000) and mouse anti PSD-95 (1:1000) in PBS for $48 \mathrm{~h}$ at $4^{\circ} \mathrm{C}$.

C: cocktail of mouse anti-TH $(1: 400)$ and rabbit antiSynI $(1: 1000)$ in PBS for $48 \mathrm{~h}$ at $4{ }^{\circ} \mathrm{C}$.

D: cocktail of rabbit anti-SynI (1:1000) and mouse anti PSD-95 (1:1000) in PBS for $48 \mathrm{~h}$ at $4^{\circ} \mathrm{C}$

All sections (for protocols B, C, D) were washed $3 \times 10 \mathrm{~min}$ in PBS, and incubated in biotinylated goat anti-mouse (1:200, Vector Laboratories, Burlingame, CA) in PBS for $2 \mathrm{~h}$ at RT. After this step, slices were incubated in a cocktail of Fluorescein-Streptavidin (Vector Laboratories, Burlingame, CA) (1:200) and anti-rabbit Alexa Fluor 546 (1:200, Molecular Probes) secondary antibodies in PBS for $2 \mathrm{~h}$ at RT and then washed $3 \times 10 \mathrm{~min}$ in PBS and coverslipped with Vectashield (Vector Laboratories, Burlingame, CA).

\section{Control procedure}

To verify the feasibility of our method, i.e. the possibility that slices impregnated as above (Golgi-Cox procedure) can be successfully used for immunofluorescence, three rats were deeply anesthetized and perfused intracardially with $0.9 \%$ saline solution as above. Brains were then immersed immediately in Golgi-Cox solution using the traditional procedure, i.e. without any post-fixation with paraformaldehyde (Fregerslev et al. 1971; Kolb and McClimans 1986; Glaser and Van der Loos 1981). Sections were collected in PBS and processed with the same immunoreactions as indicated above in the immunofluorescence procedure.

Laser scanning confocal microscopy

Slices analysis was performed using a Leica 4D confocal laser scanning microscope with an argon-krypton laser. Confocal images were generated using PL Fluotar $10 \times$ (na. $0.3), 40 \times$ oil (na. 1.00) and $100 \times$ oil (na. 1.3). Each frame was acquired eight times and then averaged to obtain noisefree images. Scans were performed in sequence using channels for fluorescein, rhodamine and reflection, using, 
exactly, the same range in $Z$-axis. Resulting datasets were combined, frame by frame, for simultaneous rendering. In this work, to detect Golgi-Cox material, the microscope was automatically set up in reflection mode by using a $488 \mathrm{~nm}$ wavelength and replacing the dichroic filter, typically used in fluorescence mode, by a 30/70 beam splitter. No barrier filter was used to direct the reflected light by the sample to the detector.

\section{Rendering}

Surface rendering was used to display and analyze the structures creating shaded solid bodies. The method, by simple thresholding, uses information from sequenced image slices using voxel elements in a topologically consistent way. A pair of voxel face hits, surrounding a vertex value exceeding the surface value are used to form a triangle. The rendered surfaces were interactively displayed and analyzed for global structure properties and interaction between fluorescence and Golgi-Cox stained elements. Maximum intensity, Extended focus, Colocalization and Simulated Fluorescence Process algorithms (Imaris 7.0) were also used.

\section{Results}

Following paraformaldehyde fixation the standard GolgiCox impregnation procedure resulted in all cases in a good staining of neural elements that were homogeneously distributed in the tissue. As a rule, only a portion of total number of neurons was casually stained. In all sections, many neurons, including their dendritic tree, dendritic spines and axons, were consistently visualized by conventional light and confocal microcopy in reflection modality. Occasionally, some astrocytes and the lumen of some capillaries were stained in a manner similar to GolgiCox alone. When the immuno procedure was applied to Golgi-Cox stained material we could appreciate very bright fluorescent elements. As expected, the penetration of antibodies was just about $25-40 \mu \mathrm{m}$ from both sides of free floating sections. Immunoreactivity was found only in sections from brains post-fixed with ice-cold $4 \%$ paraformaldehyde, while no appreciable fluorescence was found in saline perfused brains (Control rats).

\section{Mesencephalon}

\section{Golgi-Cox and TH}

Figure 1 shows both TH-positive and Golgi-Cox impregnated elements in the $\mathrm{SNc}$ simultaneously visualized by CLSM (Fig. 1a). The figure shows that the application of these two staining procedures does not affect each other since it was possible to provide information about the neurochemical feature (TH-immunolabeling alone, Fig. 1b) of impregnated neurons (impregnation alone, Fig. 1c). In addition, it was also possible to verify that the Golgi-Cox impregnation offers a more detailed representation of neuronal structures than $\mathrm{TH}$-immunolabeling procedure: in fact, as shown in panels $\mathrm{c}$ and $\mathrm{d}$ of Fig. 1. The dendrite (black arrow) that appears in panel $\mathrm{c}$ is not appreciable by $\mathrm{TH}$ fluorescence (Fig. 1b) as demonstrated by colocalization (Fig. 1d). The scansion depth in this case was of $31.5 \mu \mathrm{m}$, a distance consistently travelled by this antibody (Fig. 1f) and this supports the above interpretation. Interestingly, and in contrast with Golgi-Cox impregnation, TH-fluorescence alone (Fig. 1b) prevents the proper visualization of critical elements such as spines-like appendages demonstrated by computational analysis (Fig. 1d). The vectorial analysis shown in panel 1e, similarly, reveals that Golgi-Cox representation highlights greatly the morphological profile of cell bodies, dendrites and spines-like structures (white arrows) previously observed by Tepper et al. (1987). In particular, the analysis shown in Fig. 1e indicates that morphological assessments based exclusively on TH-immunolabeling might result in lower absolute precision.

\section{Golgi-Cox, TH and PSD-95}

The distribution of PSD-95 in the mesencephalon was not homogeneous. In fact, areas like the medial lemniscus and the basal part of cerebral peduncle, devoid of synaptic contacts, lack PSD-95 immunoreactivity, whereas the pars reticulata of the substantia nigra and the red nucleus magnocellularis reveal a greater density of PSD-95 compared to other midbrain areas (not shown). Figure 2 shows that dense punctate staining for PSD-95 was detectable in both TH-positive (green) and TH-negative Golgi-Cox impregnated neurons (red). As shown in panels b and $c$ of Fig. 2, in TH-positive neurons the PSD-95 is predominantly present corresponding to soma membrane, while in their dendrites it appears rather sparse. In the TH-negative and Golgi-Cox stained neuron the PSD-95 immunoreactivity was detected in correspondence to the soma and in the majority of dendrites. In agreement with Nowicka et al. (2003), panels a and c, reveal that such dense punctate staining of PSD-95 allows to recognize neuropils and dendrites of non-labeled cellular profiles (white arrow).

\section{Striatum}

\section{Golgi-Cox and TH}

In the striatum, many impregnated medium spiny neurons (MSN) immersed in a very large number of TH-positive fibers were observed (Fig. 3a). These fibers were very dense, 

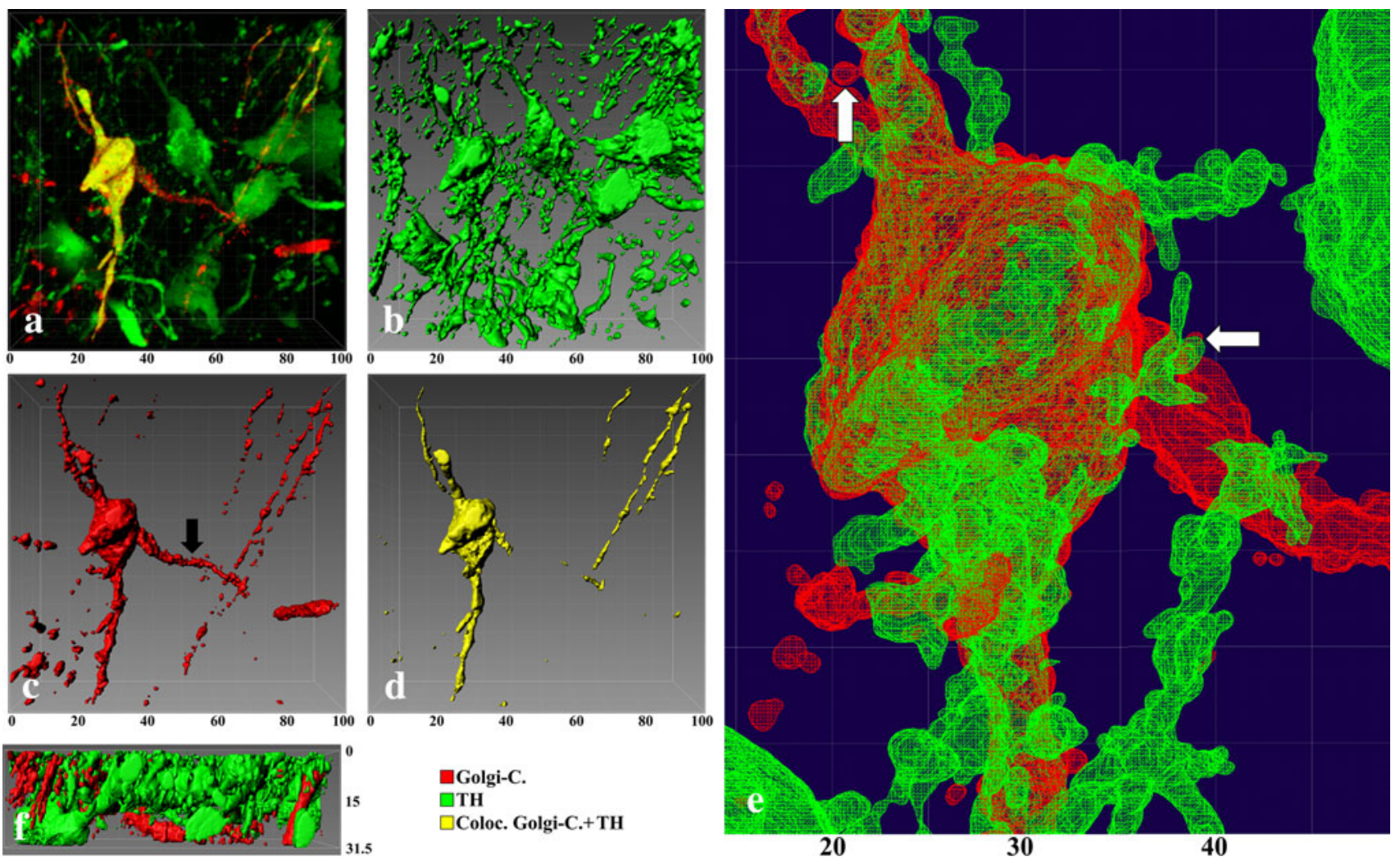

Fig. 1 Volume rendering of SNc TH-positive (green) and Golgi-Cox impregnated (red) neurons. a The rendered stack of impregnated and simultaneously immunofluorescent stained TH-positive neuron. b, c Reported the results of individual channel surface rendering for THimmunofluorescence and Golgi-Cox, respectively. The colocalization analysis (d) clearly shows that Golgi-Cox impregnation is more detailed than $\mathrm{TH}$-immunolabeling. In fact the black arrow shows a dendritic trunk not visualized in $\mathbf{d}$. It is possible to realize that the

evenly distributed in whole striatum and prevalently orientated in caudal-rostral direction. The fluorescence microscopy examination of these sections revealed that the medial forebrain bundle was particularly bright (not shown). On the contrary, in the anterior commissure, fibers were occasional or almost absent (Fig. 3a). As expected, fully impregnated MSN (visible also in white light, through the entire thickness of the specimen) showed with great details all their neuronal structures. In agreement with Freund and colleagues (1984) and Sesack and Pickel (1990), surface rendering analysis showed TH-positive terminals making prevalently putative contacts with spines' neck and dendritic shafts of MSN (Fig. 3c). Few contacts were observed between TH-positive terminals and spines heads (Fig. 2c) that testify asymmetric synapses especially with non DAergic terminals (Freund et al. 1984; Sesack and Pickel 1990).

\section{Golgi-Cox, TH, PSD-95 and SynI}

The PSD-95 and the SynI immunoreactions in the striatum were uniformly detected. When the double staining with

cellular profiles, dimension and morphology are better appreciable in Golgi-Cox versus immunofluorescence (e). The white arrows indicate some dendritic spines-like structures visible only by impregnation. This stack was also $90^{\circ}$ rotated in the $X$-axis to evaluate the penetration of anti-TH primary and secondary antibodies that are fully penetrated in scansion depth (f). Golgi-C Golgi-Cox staining; Coloc Colocalization. All scales are expressed in $\mu \mathrm{m}$

PSD-95 and SynI (in Golgi-Cox stained sections) was performed, it was possible to assess the relationship between these two antigens, MSN dendrites and dendritic spines (Fig. 4). In particular, clusters of PSD-95 were detected in association with the soma membrane of impregnated MSN and this also applied to the cell bodies profiles of non impregnated neurons as previously described relatively to mesencephalic sections (see also Fig. 2c). On the other hand, immunoreactivity of punctate SynI was always found outside impregnated and non impregnated neurons (Fig. 4a). Panels b and c of Fig. 4 demonstrate that PSD-95 colocalized within the dendrite of an impregnated MSN (yellow parts in these panels indicate colocalization between PSD-95 and Golgi-Cox impregnation). In particular, PSD-95 was present in most heads and necks of dendritic spines and frequently in the dendritic shafts. Consistently, many clusters of SynI have been identified in correspondence with the PSD-95 immunofluorescence in both spines (head and neck) and dendritic shafts thus identifying both symmetric and asymmetric synapses (Fig. 4a, d). Coherently, volume analysis revealed that 

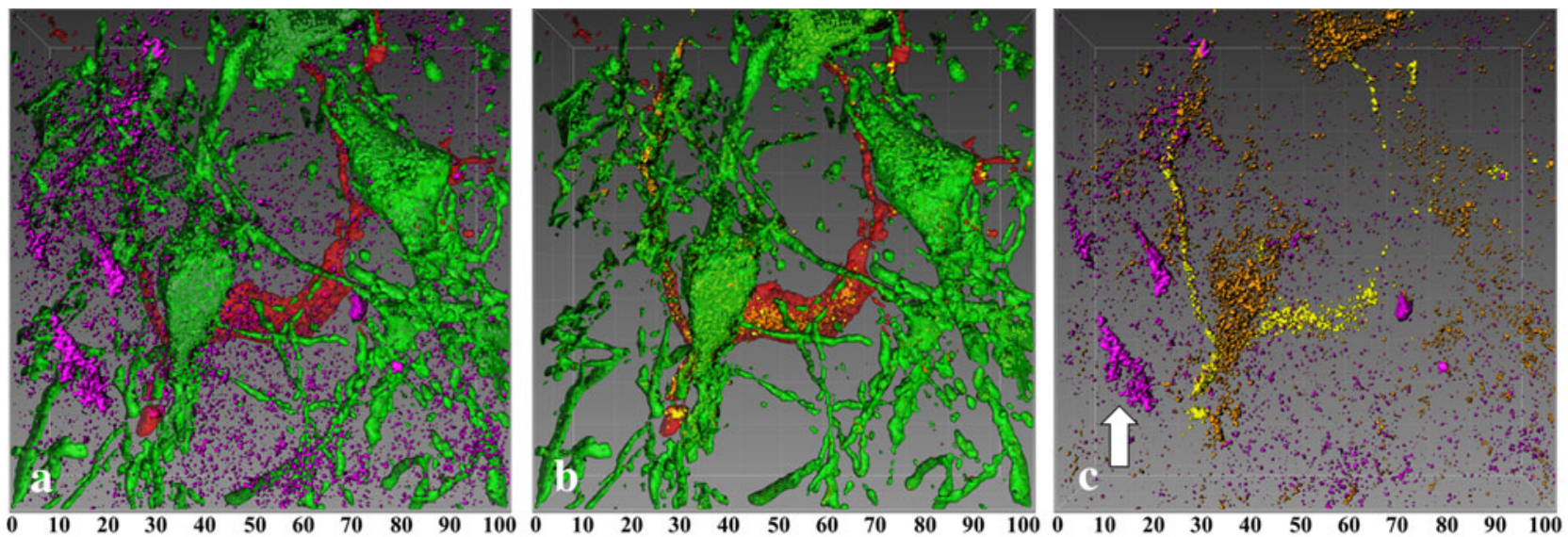

\section{$\square$ Golgi-C. $\quad \square$ PSD-95 $\square$ Coloc. TH + PSD-95 \\ $\square$ TH $\quad \square$ Coloc. Golgi-C.+ PSD-95}

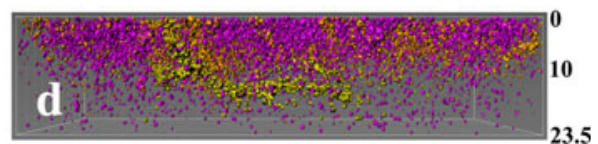

Fig. 2 Golgi-Cox impregnated (red) and TH-positive (green) neurons and punctate PSD-95 immunofluorescence revealed by surface rendering algorithm in $\mathrm{SNc}$ (a). In this picture is clearly shown the distribution of PSD-95 immunofluorescence colocalized with THpositive (orange) (b-d) Golgi-Cox impregnated (yellow) cells (b-d) and not colocalized (magenta) $(\mathbf{a}, \mathbf{c}, \mathbf{d})$. White arrow indicates a non- labeled cellular profile. To evaluate the anti PSD-95 primary and secondary antibodies penetration, the stack was rotated of $90^{\circ}$ in the $X$-axis (d). Images in $\mathbf{c}$ and $\mathbf{d}$ were obtained by unselecting detection channels for TH and Golgi-Cox. Golgi-C Golgi-Cox staining; Coloc Colocalization. All scales are expressed in $\mu \mathrm{m}$
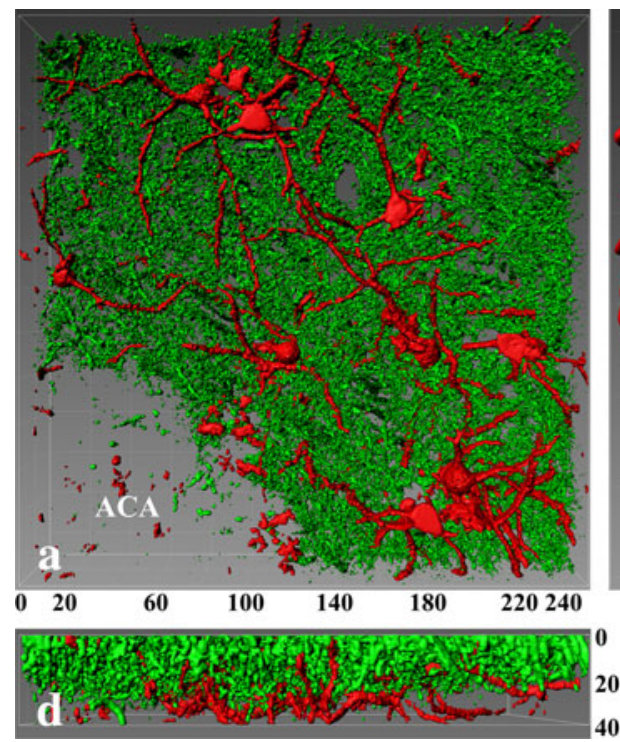

Fig. 3 Surface rendering of Golgi-Cox impregnated MSN (red) adjacent to the anterior commissure (ACA), surrounded by a dense network of TH-positive fibers (green) (a). This stack was $90^{\circ}$ rotated to show the caudo-rostral prevalent orientation of fibers (d). An

$\sim 38.6 \%$ of volume of MSN resulted covered by PSD-95 (Fig. $5 \mathrm{a}-\mathrm{c}$ ) while only $\sim 0.8 \%$ by SynI (Fig. $5 \mathrm{~d}-\mathrm{f}$ ) and only $\sim 0.6 \%$ of PSD-95 volume of MSN was found colocalized with TH-positive fibers. In addition, $\sim 22 \%$ of the volume of TH-positive terminals contained SynI aggregates (Fig. 5). accumbal shell MSN dendritic trunk (b) is enclosed in a dense network of TH-positive fibers that takes closed contacts with dendritic shaft and dendritic spines' necks (c). Golgi-C Golgi-Cox staining; Coloc Colocalization. All scales are expressed in $\mu \mathrm{m}$

Frontal Lobes

\section{Golgi Cox-TH, PSD-95 and SynI}

Various frontal regions from sections obtained from AP +3.20 to $+2.70 \mathrm{~mm}$ from bregma (Paxinos and Watson 
Fig. 4 Surface rendering of Golgi-Cox stained MSN dendritic branch (red) and double immunostaining for punctate SynI (cyan) and PSD95 (magenta) (a). The colocalization analysis shows that PSD-95 (yellow) is distributed especially into the spines head, but also into the spines neck and dendritic shaft (b-e). When joined with PSD95, SynI immunofluorescence indicates both symmetrical and asymmetrical synapses with spines' neck and head,

respectively, and dendritic trunk $(\mathbf{a}, \mathbf{e})$. Also in the striatum it was possible to detect cellular profiles of non-stained neurons (white arrow). c The same dataset rotated of $90^{\circ}$ in the $X$-axis, without SynI channel. e Some SynI puncta were manually removed to allow better understanding of the relationship between pre- and post-synaptic elements with the Golgi-Cox stained dendrite. Golgi-C Golgi-Cox staining; Coloc Colocalization. All scales are expressed in $\mu \mathrm{m}$
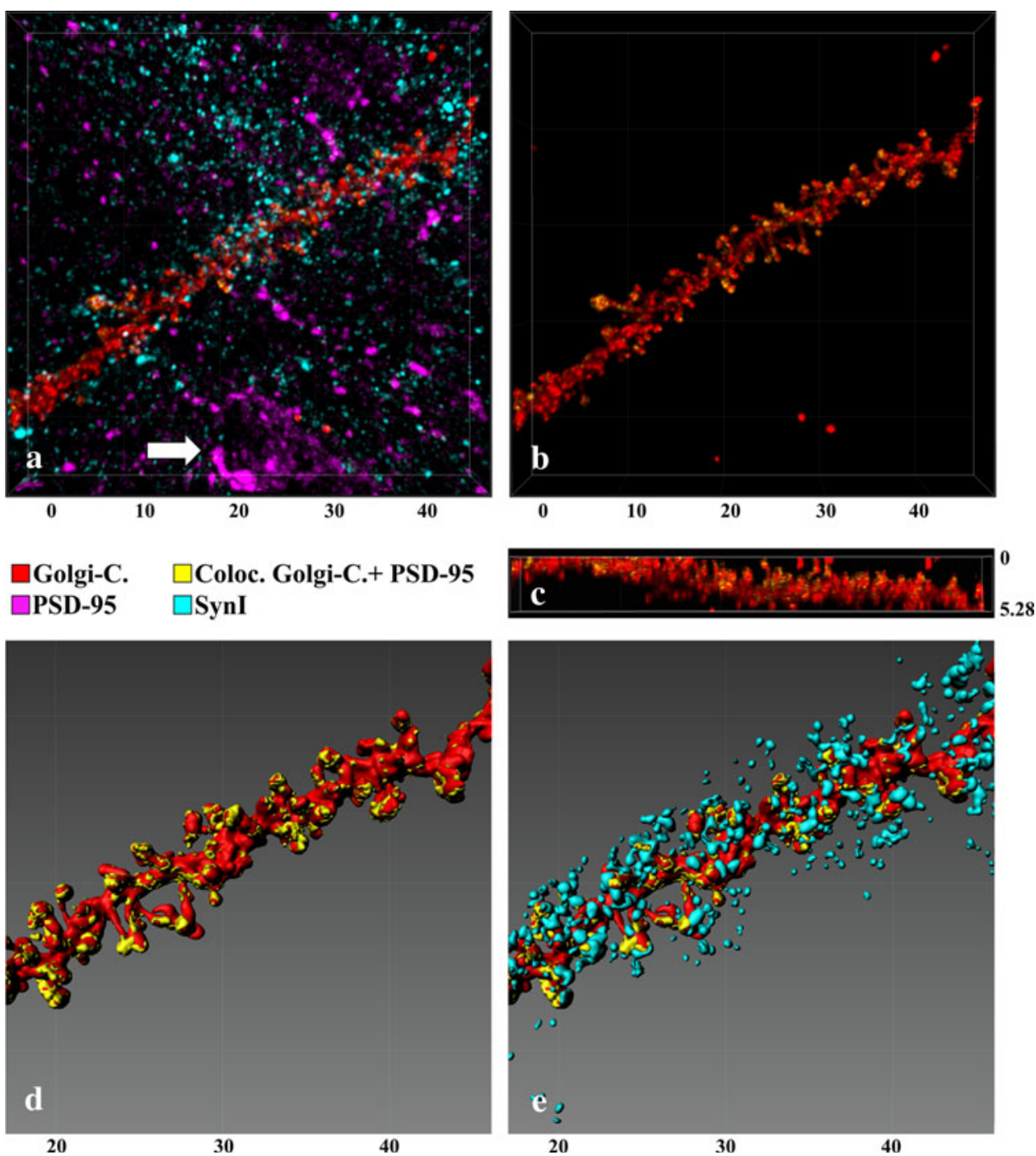

1998) have been examined to evaluate the presence and the density of TH-immunofluorescence. In the prefrontal and orbitolateral cortices the density of stained fibers was lower than that in the striatum (Fig. 6a). In layer I of the PFC THpositive fibers across, prevalently parallel to brain surface, through the distal dendrites of pyramidal neurons, while in the deepest layers of the cortex, TH-fibers run mainly perpendicular.

The intensity of fluorescence of TH-positive fibers in the olfactory tubercle (AP +3.20 to $+1.00 \mathrm{~mm}$ from bregma) was comparable to the striatum (not shown). The pyramidal and spiny neurons, in the rostral (AP $+2.2 \mathrm{~mm}$ from bregma) and caudal parts, respectively, were fully immersed in a very dense network of TH-positive fibers. Also in this case it was possible to identify putative contacts between them. In pyramidal neurons the PSD-95 immunofluorescence was strongly detected in both soma and dendritic portions (Fig. 6c, e). The distribution of SynI was uniformly detected in all layers of the cortex except for the molecular layer (Fig. 6b, d) in which it was very poor.

\section{Discussion}

The aim of the present work was to develop a simple and inexpensive method, appropriate for CLSM, to detect immunofluorescence in Golgi-Cox impregnated materials. The Golgi-Cox impregnation and immunofluorescence methods have been considered, for long time, incompatible for simultaneous application to the same sections (Lee et al. 2006). The results of the present study demonstrate that following appropriate fixation and post-fixation procedures and it is possible to obtain a biochemical characterization of morphologically identified impregnated neurons and, in addition, prevents tissue cracking on cryostat sectioning. Briefly, this method allows in 

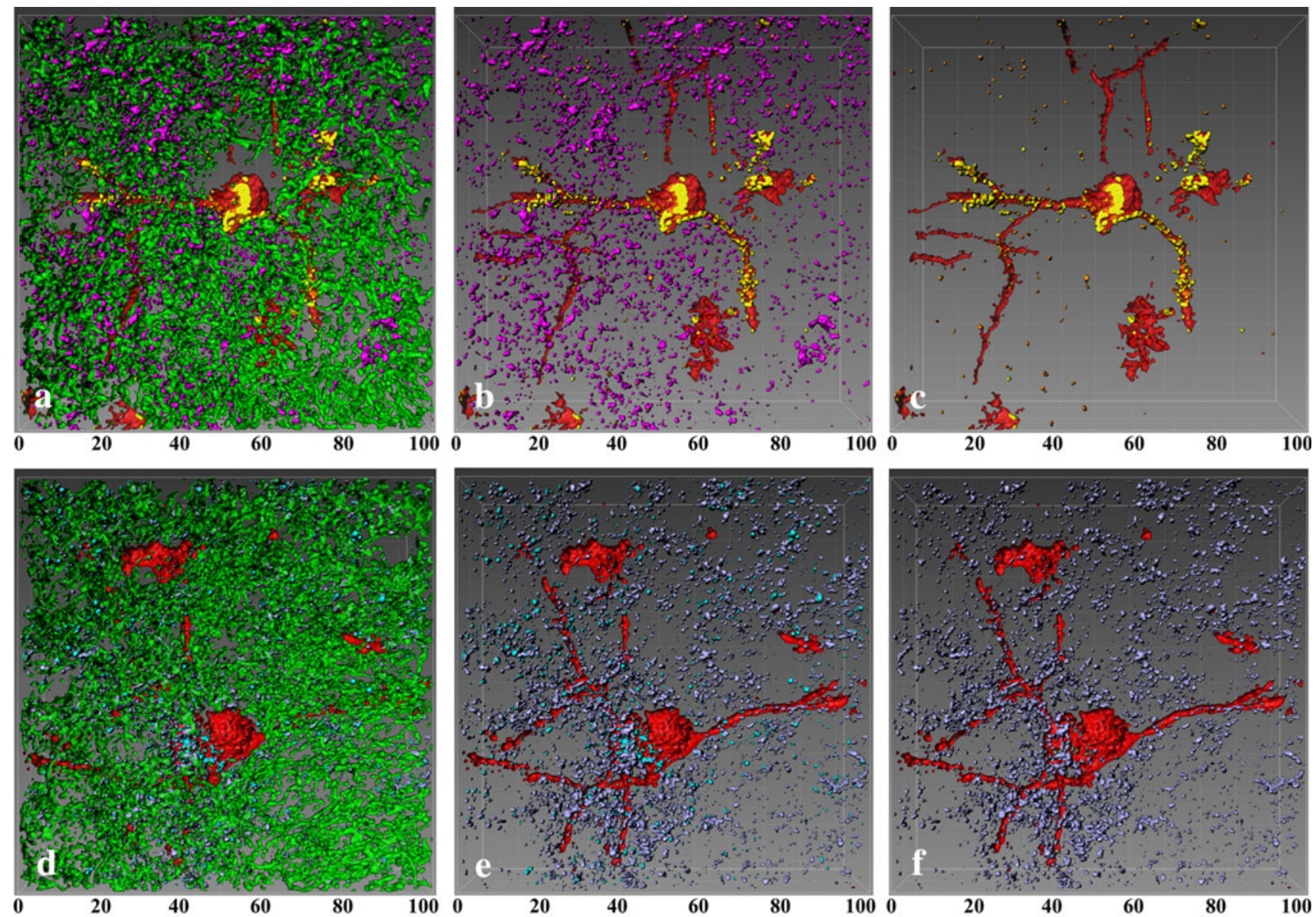

\section{口Golgi-C. $\square$ TH}

\section{$\square$ Coloc. Golgi-C.+ PSD-95 \\ $\square$ Coloc. Golgi-C.+ SynI}

\section{Coloc. TH + PSD-95 \\ $\square$ Coloc. TH + SynI}

Fig. 5 Pattern of colocalization of Golgi-Cox neuronal structures (red) (a-c) with SynI (violet) and PSD-95 (yellow) with TH (green) (d-f) immunofluorescence in the striatum. As expected, the MSN is covered by abundant PSD-95 (a-c), while colocalization analysis shows that PSD-95 (orange) is only occasionally overlapped with TH

immunofluorescent material, the detection of finest morphological details, as one would expect from Golgi-Cox impregnated tissues.

The egg of Columbus is the inverted sequential application of two fixative solutions (paraformaldehyde and Golgi-Cox solution). In fact, sections obtained from control rats, processed under a Golgi-Cox standard method (Spiga et al. 2005; Kolb and McClimans 1986) (without any fixation and post-fixation with $4 \%$ paraformaldehyde solution) allowed only the detection of impregnated neurons whereas the immunofluorescence reactions failed. This was not surprising if one considers that immunostaining techniques require a careful fixation to leave intact as many amino acid side-chains as possible. These antibody recognition sites are probably severely impaired by mercuric (b, c) immunofluoescence. SynI staining shows a very poor colocalization with MSN (blue-green) and a great superimposition with $\mathrm{TH}$ terminals (d-f). The non-colocalized PSD-95 (magenta) (a-c) and SynI (cyan) (d-f) are also shown. Golgi-C Golgi-Cox staining; Coloc Colocalization. All scales are expressed in $\mu \mathrm{m}$

chloride fixation if this is used as first fixative, although some, somewhat, successful attempts in this direction have been done previously (Buller and Rossi 1993). In fact, fixation and post fixation are critical steps in immunohistochemistry, and, especially for TH studies, $2-4 \%$ paraformaldehyde is fundamental to block the proteins that may tend to diffuse from their initial location (Berod et al. 1981). Indeed, in our study we found post-fixation to be a strategic step because the fixative-to-protein binding is slow and largely completed within $24 \mathrm{~h}$ (Helander 1994) required for the penetration of the antibodies trough slices' depth. Furthermore, even in the absence of the demanding immunohistochemical needs of the present study, the careful use of aldehydes in Golgi's methods is frequent (Moss and Whetsell 2004), especially for the study of 

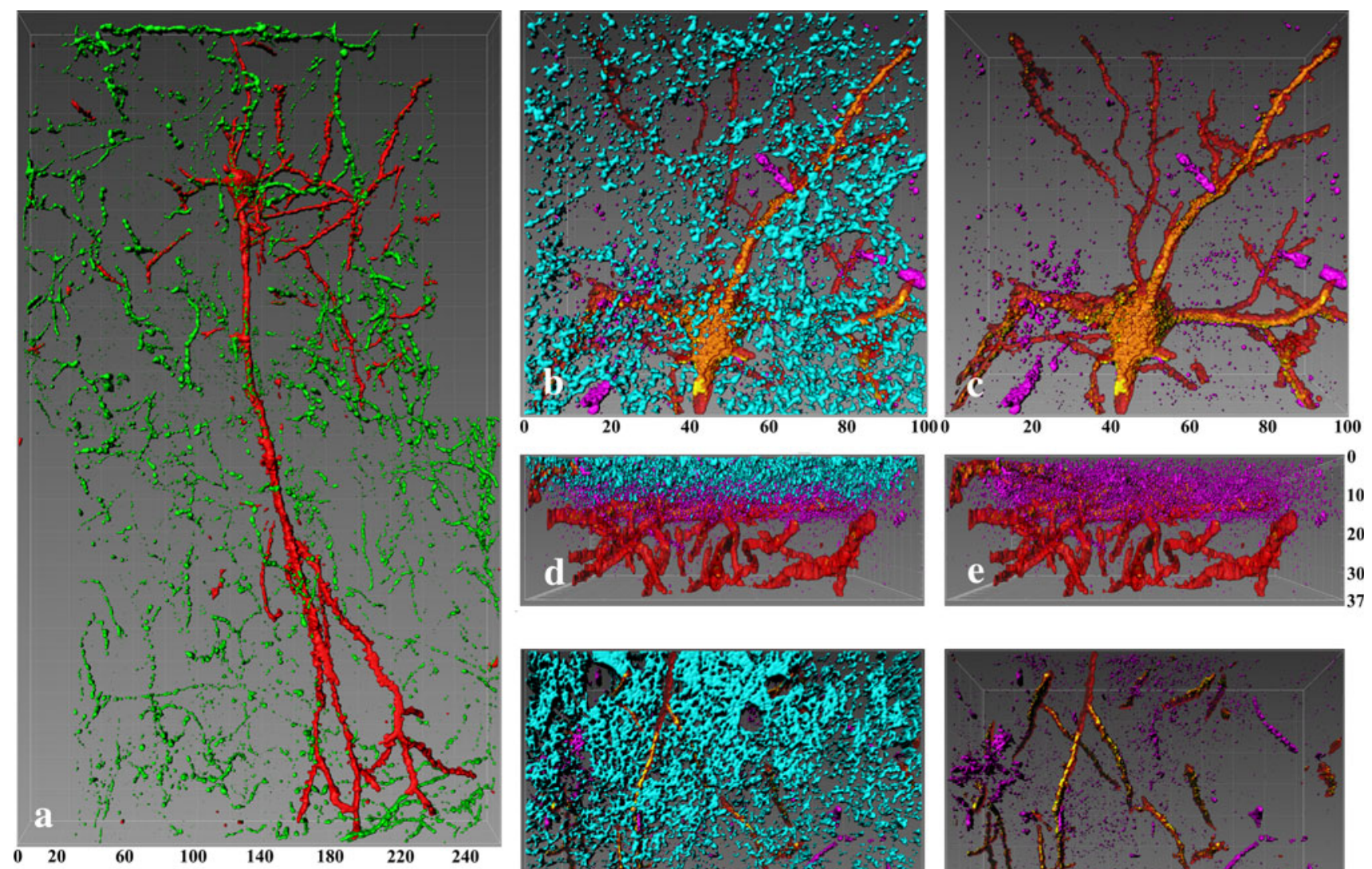

\section{口Golgi-C. $\square$ Coloc. Golgi-C.+ PSD-95
$\square$ PSD-95 $\quad \square$ SynI $\square$ TH}
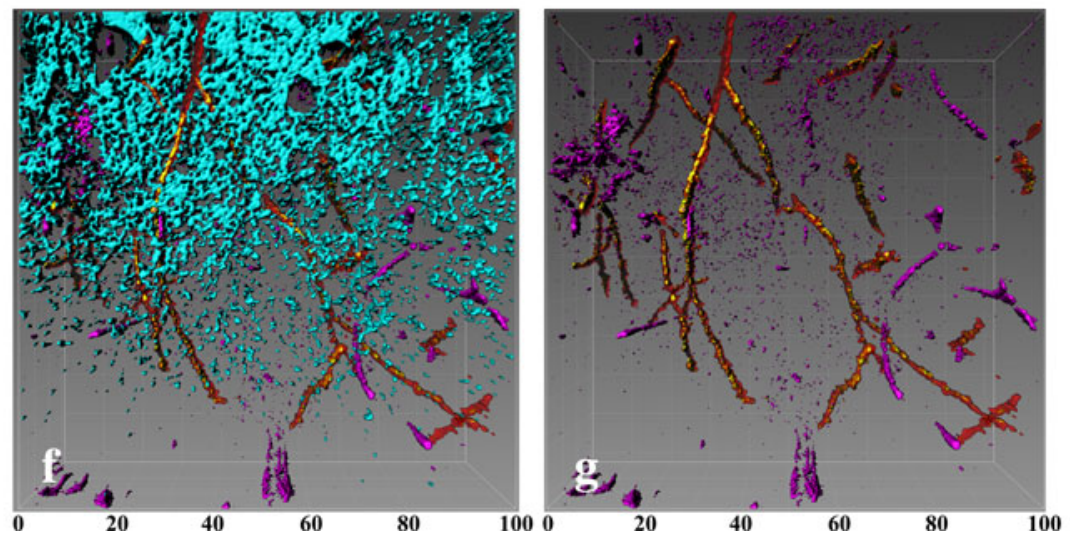

Fig. 6 Volume rendering of a Golgi-Cox stained (red) pyramidal neuron and TH-positive terminals (green) in prefrontal prelimbic zone (a). A cell body, proximal dendrites (b-e) in layer 4 and distal dendrites in layers 1 and 2 (f, $\mathbf{g}$ ) show the same relationship with preand post-synaptic proteins than for MSN in the striatum. In particular, the PSD-95 immunofluorescence is present (yellow) in the soma and in both proximal and distal dendrites located in the first 20-25 $\mu \mathrm{m}$ of the slice depth (d, e), while the anti-SynI (cyan) penetrates for $10-15 \mu \mathrm{m}$ in the Z-axis (d). Interestingly, the SynI immunofluorescence is almost absent in molecular layer 1 of the cortex (f). Golgi-C Golgi-Cox staining; Coloc Colocalization. All scales are expressed in $\mu \mathrm{m}$

vein, Buller and Rossi (1993) showed that some antigens could still be localized, with immunohistochemical methods, after Golgi-Cox fixation. Furthermore, these authors have also tested many combinations of antibodies with Golgi-Cox fixation until the case in which, in order to distinguish immunoperoxidase labeled structures, the mercuric deposit from specimens was removed because it could cover brown diaminobenzidine tetrahydrochloride labeled structures. Our method, instead, obviates these difficulties and, indeed, transforms problems and/or limitations into advantages. In particular, as shown in Fig. 1, we were able to separately visualize in its finest anatomical details a TH-positive immunofluorescent neuron. Finally, the application of 3D reconstruction and vectorization algorithms permitted us to make reliable and detailed 
pictures to study the localization/colocalization and to determine the spatial and anatomical relationships among stained elements.

To test this procedure we sampled various brain regions along the DAergic pathway, from midbrain to PFC. The use of anti-TH and pre- and post-synaptic markers, provided informations about the distribution and relationship of the relative antigens within neurons variously stained. Thus, the use of anti PSD-95 shows a punctate appearance that was, in accordance with Yoshii et al. (2003), mostly localized in perinuclear cytoplasm and dendrites. As expected the PSD-95 puncta were found, in MSN dendrites, localized post-synaptically in the spines' neck, head (Zhang et al. 2007) and dendritic trunks. Interestingly, the punctate labeling pattern is characteristic of PSD-95 staining at synapses (Yoshii et al. 2003) and besides the perinuclear cytoplasm the presence of PSD-95 immunolabeling is frequently described in the literature both in vivo and in vitro (Yoshii et al. 2003; Zhang et al. 2007).

Not surprisingly, the density of TH-positive terminals in the prefrontal cortex was found lesser as compared to the striatum. This aspect is particularly highlighted in Figs. 3, 5 and 6 that show the relationship between dopaminoceptive neuronal structures (i.e., in particular dendritic spines) and DAergic fibers (TH-positive) probably arising from the mesencephalon. In addition, Fig. 3 details TH-positive terminals that converge in dendritic spines of MSN DAceptive neurons as previously described (Freund et al. 1984; Goldman-Rakic et al. 1989; Carr and Sesack 1996). Interestingly, the great majority of spines' heads were devoid of contacts with TH-positive fibers and this might be related with the different nature of fibers that synapse on spines' heads. In conclusion the present paper shows that the combination of these two very different methods, combined for long time to be incompatible, can be considered into an innovative tool to yield finest morphological detail with neurochemical identity of nerve cells.

Facts or artifacts?

In microscopy, an artifact is an apparent structural detail introduced by the processing that does not concern specimen's features. In particular, in confocal microscopy, the bleed-through (also termed crosstalk or crossover) artifacts often complicate the interpretation of experimental results, and in particular the subcellular colocalization of fluorophores. This phenomenon is usually manifested by the emission of one fluorophore through the filter reserved for a second fluorophore. For example, it is know that in double labeling with the traditional fluorescence in rhodamine probes, the bleed-through can only be reduced but is never completely eliminated. In this work the problem of crosstalk was meticulously looked on, because to our knowledge no data have been previously published about possible bleed-through between Golgi-Cox stained material and fluorescent probes. Indeed, in some cases we have observed that the "black reaction", with high power of laser in reflection modality, emitted through the filter for rhodamine. However, although we could not dismiss the possibility of artifacts, a number of reasons suggest that these were minor and/or negligible. First, the pattern of distribution of immunoreactivity of TH, as well as of PSD95 and Syn-I, were in agreement with previous immunofluorescent-only preparation studies (Nowicka et al. 2003; Seifert et al. 1998; Nikonenko et al. 2008). Second, the CLSM analysis allows to detect and measure the degree of penetration through the slices' depth (Z-axis) of the antibodies in the slices. In fact, tissue penetration of antibody depends on target density, antibody affinity and diffusion characteristics of the antibody and on fixation procedures. Despite the use of high concentrations of Triton X-100 and long incubation times, antibody penetration into slices is poor (Adermark and Lovinger 2006). By CLSM, we were able to assess that antibodies could penetrate the slices by no more than 35-40 $\mathrm{m}$ for TH (Figs. 1f, 3d) and 25-30 $\mu \mathrm{m}$ for SynI (Fig. 6d) and PSD-95 (Figs. 2d, 6d, e). In contrast, the Golgi-Cox impregnation penetrates slices thoroughly (Figs. 1f, 3d, 6d, e). In particular, whereas THimmunofluorescence in the mesencephalon, striatum and cortex cannot be mistaken as an artifact, the attribution of punctate SynI and PSD-95 immunofluorescence to neuronal structures might result more difficult. Figure $6 \mathrm{~b}, \mathrm{c}, \mathrm{f}, \mathrm{g}$ shows that whereas Golgi-Cox material extends through the slice's depth, the immunoreactivity of PSD-95, colocalized within the neuron, is unmistakably distributed only to soma and dendrites within the first $27.5 \mu \mathrm{m}$ from the slice's surface. We interpret this observation as a proof of the fact that it is not the presence of Golgi-Cox material in the slice that generates this signal.

Acknowledgments This work was supported, in part, by a grant from the Compagnia di San Paolo, project no. 3906 to E.A. and MIUR (PRIN. No. 2004052392 and 2006057754) to M.D.

Conflict of interest The authors declare no competing financial interests.

Open Access This article is distributed under the terms of the Creative Commons Attribution Noncommercial License which permits any noncommercial use, distribution, and reproduction in any medium, provided the original author(s) and source are credited.

\section{References}

Adermark L, Lovinger DM (2006) Ethanol effects on electrophysiological properties of astrocytesin striatal brain slices. Neuropharmacology 51:1099-1108 
Berod A, Hartman BK, Pujol JF (1981) Importance of fixation in immunohistochemistry: use of formaldehyde solutions at variable $\mathrm{pH}$ for the localization of tyrosine hydroxylase. J Histochem Cytochem 29(7):844-850

Buell SJ (1982) Golgi-Cox and rapid Golgi methods as applied to autopsied human brain tissue: widely disparate results. J Neuropathol Exp Neurol 41(5):500-507

Buller JR, Rossi ML (1993) Immunocytochemistry on paraffin wax Golgi-Cox impregnated central nervous tissue. Funct Neurol 8(2):135-151

Carr DB, Sesack SR (1996) Hippocampal afferents to the rat prefrontal cortex: synaptic targets and relation to dopamine terminals. J Comp Neurol 369(1):1-15

Colonnier M (1964) The tangential organization of the visual cortex. J Anat 98:327-344

Cox W (1891) Impragnation des centralen Nervensystems mit Quecksilbersalzen. Arch Mikr Anat 98:327

Diana M, Spiga S, Acquas E (2006) Persistent and reversible morphine withdrawal-induced morphological changes in the nucleus Accumbens. Ann N Y Acad Sci 1074:446-457

Flores C, Wen X, Labelle-Dumais C, Kolb B (2007) Chronic phencyclidine treatment increases dendritic spine density in prefrontal cortex and nucleus accumbens neurons. Synapse 61(12):978-984

Fregerslev S, Blackstad TW, Fredens K, Holm MJ, Ramón-Moliner E (1971) Golgi impregnation with mercuric chloride: studies on the precipitate by X-ray powder diffraction and selected area electron diffraction. Histochemie 26(4):289-304 (1971)

Freund TF, Somogyi P (1983) The section-Golgi impregnation procedure. 1 Description of the method and its combination with histochemistry after intracellular iontophoresis or retrograde transport of horseradish peroxidase. Neuroscience 9(3):463-474

Freund TF, Powell JF, Smith AD (1984) Tyrosine hydroxylaseimmunoreactive boutons in synaptic contact with identified striatonigral neurons, with particular reference to dendritic spines. Neuroscience 13(4):1189-1215

Friedland DR, Los JG, Ryugo DK (2006) A modified Golgi staining protocol for use in the human brain stem and cerebellum. J Neurosci Methods 150(1):90-95

Glantz LA, Lewis DA (2000) Decreased dendritic spine density on prefrontal cortical pyramidal neurons in schizophrenia. Arch Psychiatry 57:65-73

Glaser EM, Van der Loos H (1981) Analysis of thick brain sections by obverse-reverse computer microscopy: application of a new, high clarity Golgi-Nissl stain. J Neurosci Methods 4(2):117-125

Goldman-Rakic PS, Leranth C, Williams SM, Mons N, Geffard M (1989) Dopamine synaptic complex with pyramidal neurons in primate cerebral cortex. Proc Natl Acad Sci USA 86(22):9015-9019

Golgi C (1873) Sulla struttura della sostanza grigia del cervello. Gaz. Med. Lombarda 33:244-246

Gorazd R, Mancevski B, Ilievski B, Perera T, Lisanby SH, Coplan JD, Duma A, Serafimova T, Dwork AJ (2003) Optimization of Golgi methods for impregnation of brain tissue from humans and monkeys. J Neurosci Methods 131(1-2):1-7

Helander KG (1994) Kinetic studies of formaldehyde binding in tissue. Biotech Histochem 69(3):177-179

Kasture S, Vinci S, Ibba F, Puddu A, Marongiu M, Murali B, Pisanu A, Lecca D, Zernig G, Acquas E (2009) Withania somnifera prevents morphine withdrawal-induced decrease in spine density in nucleus accumbens shell of rats: a confocal laser scanning microscopy study. Neurotox Res 16(4):343-355

Kolb B, McClimans J (1986) Cryostat sectioning of Golgi-Cox tissue. Stain Technol 61(6):379-380

Kolb B, Cioe J, Comeau W (2008) Contrasting effects of motor and visual spatial learning tasks on dendritic arborization and spine density in rats. Neurobiol Learn Mem 90(2):295-300
Kopsch F (1896) Erfahrungen über die Verwendung des Formaldehyds bei der Chromsilber-Imprägnation. Anat Anz 11:727

Lee KW, Kim Y, Kim AM, Helmin K, Nairn AC, Greengard P (2006) Cocaine-induced dendritic spine formation in D1 and D2 dopamine receptor-containing medium spiny neurons in nucleus accumbens. Proc Natl Acad Sci USA 103(9):3399-3404

Moss TL, Whetsell WO (2004) Techniques for thick-section Golgi impregnation of formalin-fixed brain tissue methods Mol Biol 277:277-285

Muller M (2002) Introduction to confocal fluorescence microscopy. Shaker Publishing, Maastricht

Nikonenko I, Boda B, Steen S, Knott G, Welker E, Muller D (2008) PSD-95 promotes synaptogenesis and multi innervated spine formation through nitric oxide signaling. J Cell Biol 183(6):1115-1127

Nowicka D, Liguz-Lecznar M, Skangiel-Kramska J (2003) A surface antigen delineating a subset of neurons in the primary somatosensory cortex of the mouse. Acta Neurobiol Exp 63(3):185-195

Paxinos G, Watson C (1998) The rat brain in stereotaxic coordinates, 3rd edn. Sydney, Academic Press

Rema V, Bali KK, Ramachandra R, Chugh M, Darokhan Z, Chaudhary R (2008) Cytidine-5-diphosphocholine supplement in early life induces stable increase in dendritic complexity of neurons in the somatosensory cortex of adult rats. Neuroscience 155(2):556-564

Robinson TE, Kolb B (1999) Alterations in the morphology of dendrites and dendritic spines in the nucleus accumbens and prefrontal cortex following repeated treatment with amphetamine or cocaine. Eur J Neurosci 11(5):1598-1604

Rosoklija G, Toomayan G, Ellis SP, Keilp J, Mann JJ, Latov N, Hays AP, Dwork AJ (2000) Structural abnormalities of subicular dendrites in subjects with schizophrenia and mood disorders: preliminary findings. Arch Psychiatry 57:349-356

Sarti F, Borgland SL, Kharazia VN, Bonci A (2007) Acute cocaine exposure alters spine density and long-term potentiation in the ventral tegmental area. Eur J Neurosci 26(3):749-756

Seifert U, Härtig W, Grosche J, Brückner G, Riedel A, Brauer K (1998) Axonal expression sites of tyrosine hydroxylase, calretinin- and calbindin-immunoreactivity in striato-pallidal and septal nuclei of the rat brain: a double-immunolabeling study. Brain Res 795:1-2

Sesack SR, Pickel VM (1990) In the rat medial nucleus accumbens, hippocampal and catecholaminergic terminals converge on spiny neurons and are in opposition to each other. Brain Res 527(2):266-279

Somogyi P (1990) Synaptic connections of neurones identified by Golgi impregnation: characterization by immunocytochemical, enzyme histochemical, and degeneration methods. J Electron Microsc Tech 15(4):332-351

Somogyi P, Freund TF, Wu JY, Smith AD (1983) The section-Golgi impregnation procedure. 2 Immunocytochemical demonstration of glutamate decarboxylase in Golgi-impregnated neurons and in their afferent synaptic boutons in the visual cortex of the cat. Neuroscience 9(3):475-490

Speacek J (1989) Dynamics of the Golgi method a time lapse study of early stages of impregnation in single sections. J Neurocytol 18:27-38

Spiga S, Serra GP, Puddu MC, Foddai M, Diana M (2003) Morphine withdrawal-induced abnormalities in the VTA: confocal laser scanning microscopy. Eur J Neurosci 17(3):605-612

Spiga S, Puddu MC, Pisano M, Diana M (2005) Morphine withdrawal-induced morphological changes in the nucleus accumbens. Eur J Neurosci 22(9):2332-2340

Stean JP (1974) Some evidence of the nature of the Golgi-Cox deposit and its biochemical origin. Histochemistry 40(4):377-383 
Tepper JM, Sawyer SF, Groves PM (1987) Electrophysiologically identified nigral dopaminergic neurons intracellularly labeled with HRP: light-microscopic analysis. J Neurosci 7(9):2794-2806

Williams RS (1983) Golgi's method of staining nerve cells. J Neuropathol Exp Neurol 42:210-212

Yoshii A, Sheng MH, Constantine-Paton M (2003) Eye opening induces a rapid dendritic localization of PSD-95 in central visual neurons. Proc Natl Acad Sci USA 100(3):1334-1339
Zhang J, Vinuela A, Neely MH, Hallett PJ, Grant SG, Miller GM, Isacson O, Caron MG, Yao WD (2007) Inhibition of the dopamine D1 receptor signaling by PSD-95. J Biol Chem 282(21):15778-15789 